\title{
RELATION ON SOME SUMMABILITY METHODS
}

\author{
W. T. SULAIMAN
}

\begin{abstract}
In this note a new theorem concerning $\varphi-|C, \alpha|_{k}$ summability of infinite series is proved. This Theorem contains as a special case the result of Bor (1986) which gives a relation between the two summability methods $\left|\bar{N}, p_{n}\right|_{k}$ and $|C, 1|_{k}$.
\end{abstract}

\section{Introduction}

Let $A=\left(a_{n k}\right)$ be an infinite matrix of complex numbers $a_{n k}(n, k=1,2, \cdots)$ and let $\left(\varphi_{n}\right)$ be a sequence of complex numbers. Let $\sum a_{n}$ be a given infinite series with sequence of partial sums $\left(s_{n}\right)$. We denote by $A_{n}(s)$ the $A$-transform of the sequence $s=\left(s_{r}\right)$,

$$
A_{n}(s)=\sum_{r=1}^{\infty} a_{n r} s_{r}
$$

We say that the series $\sum a_{n}$ is summable $|A|$, if

$$
\sum_{n=1}^{\infty}\left|A_{n}(s)-A_{n-1}(s)\right|<\infty .
$$

and it is said to be summable $\varphi-|A|_{k}, k \geq 1$, if (see [1])

$$
\sum_{n=1}^{\infty}\left|\varphi_{n}\left[A_{n}(s)-A_{n-1}(s)\right]\right|^{k}<\infty
$$

If we take $\varphi_{n}=n^{1-1 / k}$ (resp. $\varphi_{n}=n^{\delta+1-1 / k}$, where $\delta \geq 0$ ), then $\varphi-|A|_{k}$ summability is the same as $|A|_{k}$ (resp. $|A, \delta|_{k}$ ) summability (see [4], [5]).

Received March 10, 1993; revised August 2, 1993.

1991 Mathematics Subject Classification. 40C05, 40F05.

Key words and phrases. Summability methods, infinite series, absolutely summable, $\varphi-|C, \alpha|_{k}$ summability. 
Let $\sigma_{n}^{\delta}$ and $\eta_{n}^{\delta}$ denote the $n$-th Cesàro mean of order $\delta(\delta>-1)$ of the sequences $\left(s_{n}\right)$ and $\left(n a_{n}\right)$ respectively. The series $\sum a_{n}$ is said to be absolutely summable $(C, \delta)$ with index $k$, or simply summable $|C, \delta|_{k}, k \geq 1$, if

$$
\sum_{n=1}^{\infty} n^{k-1}\left|\sigma_{n}^{\delta}-\sigma_{n-1}^{\delta}\right|^{k}<\infty
$$

or equivalently

$$
\sum_{n=1}^{\infty} \frac{1}{n}\left|\eta_{n}^{\delta}\right|^{k}<\infty
$$

Let $\left(p_{n}\right)$ be a sequence of positive real constants such that

$$
P_{n}=\sum_{v=0}^{n} p_{v} \rightarrow \infty \quad \text { as } \quad n \rightarrow \infty\left(P_{-1}=p_{-1}=0\right) \text {. }
$$

The series $\sum a_{n}$ is said to be summable $\left|\bar{N}, p_{n}\right|_{k}, k \geq 1$, if

$$
\sum_{n=1}^{\infty}\left(\frac{P_{n}}{p_{n}}\right)^{k-1}\left|T_{n}-T_{n-1}\right|^{k}<\infty \quad \text { (see [2]) }
$$

where

$$
T_{n}=\frac{1}{P_{n}} \sum_{v=0}^{n} p_{v} s_{v}
$$

If we take $p_{n}=1$, then $\left|\bar{N}, p_{n}\right|_{k}$ summability is equivalent to $|C, 1|_{k}$ summability. $\left|\bar{N}, p_{n}\right|_{1}$ is the same as $\left|\bar{N}, p_{n}\right|$. In general the two methods $|C, \delta|_{k}$ and $\left|\bar{N}, p_{n}\right|_{k}$ are not comparable.

Bor (1986) established the following result.

Theorem A. Let $\left(p_{n}\right)$ be a sequence of positive real constants such that as $n \rightarrow \infty$

$$
\text { (i) } n p_{n}=O\left(P_{n}\right), \quad \text { (ii) } P_{n}=O\left(n p_{n}\right) \text {. }
$$

If $\sum a_{n}$ is summable $\left|\bar{N}, p_{n}\right|_{k}$, then it is summable $|C, 1|_{k}, k \geq 1$.

\section{Main Result}

We prove the following

Theorem B. Let $\left(\chi_{n}\right)$ be a positive non-decreasing sequence and let $\left(\beta_{n}\right)$ and $\left(\lambda_{n}\right)$ be sequences such that

$$
\begin{gathered}
\left|\triangle \lambda_{n}\right| \leq \beta_{n} \\
\beta_{n} \rightarrow 0 \text { as } n \rightarrow \infty
\end{gathered}
$$




$$
\begin{gathered}
\sum_{n=1}^{\infty} n\left|\triangle \beta_{n}\right| \chi_{n}<\infty \\
\left|\lambda_{n}\right| \chi_{n}=O(1) .
\end{gathered}
$$

If there exists an $\epsilon>0$ such that the sequence $\left(n^{\epsilon-k}\left|\varphi_{n}\right|^{k}\right)$ is non-increasing and

$$
\sum_{v=1}^{n} v^{1-k \alpha}\left(\frac{P_{v}}{p_{v}}\right)^{k-1}\left|\varphi_{v} \Delta T_{v-1}\right|^{k}=O\left(\chi_{n}\right), n \rightarrow \infty
$$

where $\left(p_{n}\right)$ is a sequence of positive real constants satisfying (I), and $T_{n}$ is the $\left(\bar{N}, p_{n}\right)$-mean of the series $\sum a_{n}$, then the series $\sum a_{n} \lambda_{n}$ is summable $\varphi-$ $|C, \alpha|_{k}, k \geq 1,1-1 / k<\alpha \leq 1$.

Remark. If we put $\alpha=1, \lambda_{n}=1, \varphi_{v}=v^{1-1 / k}$, and $\chi_{n}=1$ in Theorem $B$, we obtain Theorem $A$.

\section{Lemmas}

Lemma. 1[6]. If the conditions (2.1)-(2.4) are satisfied, then

$$
n \beta_{n} \chi_{n}=O(1)
$$

and

$$
\sum_{n=1}^{\infty} \beta_{n} \chi_{n}<\infty
$$

Lemma 2[7]. If $\sigma>\delta>0$, then

$$
\sum_{n=v+1}^{m} \frac{(n-v)^{\delta-1}}{n^{\sigma}}=O\left(v^{\delta-\sigma}\right), \quad m \rightarrow \infty
$$

\section{Proof of Theorem $B$}

Let $t_{n}^{\alpha}$ be the $n$-th $(C, \alpha)$-mean of the sequence $\left(n a_{n} \lambda_{n}\right)$. Then in order to prove the Theorem, it is sufficient to show that

$$
\sum_{n=1}^{\infty} \frac{1}{n^{k}}\left|\varphi_{n} t_{n}^{\alpha}\right|^{k}<\infty
$$

where

$$
\begin{aligned}
& t_{n}^{\alpha}=\frac{1}{A_{n}^{\alpha}} \sum_{v=1}^{n} A_{n-v}^{\alpha-1} v a_{v} \lambda_{v} \\
& A_{n}^{\alpha}=\left(\begin{array}{c}
n+\alpha \\
n
\end{array}\right)=\frac{(\alpha+1)(\alpha+2) \cdots(\alpha+n)}{n !} \simeq \frac{n^{\alpha}}{\Gamma(\alpha+1)} \text {. }
\end{aligned}
$$


As

$$
T_{n}=\frac{1}{P_{n}} \sum_{v=0}^{n} p_{v} \sum_{r=0}^{v} a_{r}=\frac{1}{P_{n}} \sum_{v=0}^{n}\left(P_{n}-P_{v-1}\right) a_{v}
$$

then

$$
T_{n}-T_{n-1}=\frac{p_{n}}{P_{n} P_{n-1}} \sum_{v=1}^{n} P_{v-1} a_{v}
$$

A.bel's transformation gives

$$
\begin{aligned}
t_{n}^{\alpha}= & \frac{1}{A_{n}^{\alpha}} \sum_{v=1}^{n} A_{n-v}^{\alpha-1} v a_{v} \lambda_{v}=\frac{1}{A_{n}^{\alpha}} \sum_{v=1}^{n} P_{v-1} a_{v}\left\{v A_{n-v}^{\alpha-1} P_{v-1}^{-1} \lambda_{v}\right\} \\
= & \frac{1}{A_{n}^{\alpha}}\left[\sum_{v=1}^{n-1}\left(\sum_{r=1}^{v} P_{r-1} a_{r}\right) \Delta\left(v A_{n-v}^{\alpha-1} P_{v-1}^{-1} \lambda_{v}\right)+\left(\sum_{r=1}^{n} P_{r-1} a_{r}\right) n P_{v-1}^{-1} \lambda_{n}\right] \\
= & \frac{1}{A_{n}^{\alpha}}\left[\sum _ { v = 1 } ^ { n - 1 } \left\{v \frac{P_{v}}{p_{v}} \Delta A_{n-v}^{\alpha-1} \lambda_{v} \Delta T_{v-1}+v A_{n-v-1}^{\alpha-1} \lambda_{v} \Delta T_{v-1}-\frac{P_{v-1}}{p_{v}} A_{n-v-1}^{\alpha-1} \lambda_{v} \Delta T_{v-1}\right.\right. \\
& \left.\left.+(v+1) \frac{P_{v-1}}{p_{v}} A_{n-v-1}^{\alpha-1} \Delta \lambda_{v} \Delta T_{v-1}\right\}+n \frac{P_{n}}{p_{n}} \lambda_{n} \Delta T_{n-1}\right] \\
= & t_{n, 1}^{\alpha}+t_{n, 2}^{\alpha}+t_{n, 3}^{\alpha}+t_{n, 4}^{\alpha}+t_{n, 5}^{\alpha}, \text { say. }
\end{aligned}
$$

In order to prove the Theorem, it is sufficient, by Minkowski's inequality, to show that

$$
\sum_{n=1}^{\infty} \frac{1}{n^{k}}\left|\varphi_{n} t_{n, j}^{\alpha}\right|^{k}<\infty, \quad j=1,2,3,4,5 .
$$

Applying Hölder's inequality, we have

$$
\begin{aligned}
& \sum_{n=2}^{m+1} \frac{1}{n^{k}}\left|\varphi_{n} t_{n, 1}^{\alpha}\right|^{k} \\
\leq & \sum_{n=2}^{m+1} \frac{\left|\varphi_{n}\right|^{k}}{n^{k}\left(A_{n}^{\alpha}\right)^{k}} \sum_{v=1}^{n-1} v^{k}\left(\frac{P_{v}}{p_{v}}\right)^{k}\left|\Delta A_{n-v}^{\alpha-1}\right|\left|\lambda_{v}\right|^{k}\left|\Delta T_{v-1}\right|^{k}\left\{\sum_{v=1}^{n-1}\left|\Delta A_{n-v}^{\alpha-1}\right|\right\} \\
= & O(1) \sum_{n=2}^{m+1} \frac{\left|\varphi_{n}\right|^{k}}{n^{k+k \alpha}} \sum_{v=1}^{n-1} v^{k}\left(\frac{P_{v}}{p_{v}}\right)^{k}(n-v)^{\alpha-2}\left|\lambda_{v}\right|^{k}\left|\Delta T_{v-1}\right|^{k}\left\{\sum_{v=1}^{n-1}(n-v)^{\alpha-2}\right\}^{k-1} \\
= & O(1) \sum_{v=1}^{m} v^{k}\left(\frac{P_{v}}{p_{v}}\right)^{k}\left|\lambda_{v}\right|^{k}\left|\triangle T_{v-1}\right|^{k} \sum_{n=v+1}^{m+1} \frac{\left|\varphi_{n}\right|^{k}(n-v)^{\alpha-2}}{n^{k+k \alpha}}, 0<\alpha<1, \\
& \left(\text { when } \alpha=1, t_{n, 1}^{\alpha}=0, \text { as } \triangle A_{n-v}^{\alpha-1}=0\right) \\
= & O(1) \sum_{v=1}^{m} v^{-k \alpha}\left(\frac{P_{v}}{p_{v}}\right)^{k}\left|\lambda_{v}\right|^{k}\left|\varphi_{v}\right|^{k}\left|\Delta T_{v-1}\right|^{k} \sum_{n=v+1}^{m+1} \frac{1}{(n-v)^{2-\alpha}}
\end{aligned}
$$




$$
\begin{aligned}
& =O(1) \sum_{v=1}^{m}\left|\lambda_{v}\right| v^{1-k \alpha}\left(\frac{P_{v}}{p_{v}}\right)^{k-1}\left|\varphi_{v} \Delta T_{v-1}\right|^{k} \\
& =O(1) \sum_{v=1}^{m-1} \Delta\left|\lambda_{v}\right| \chi_{v}+O(1)\left|\lambda_{m}\right| \chi_{m} \\
& =O(1) \sum_{v=1}^{m-1}\left|\Delta \lambda_{v}\right| \chi_{v}+O(1)\left|\lambda_{m}\right| \chi_{m} \\
& =O(1) \sum_{v=1}^{m} \beta_{v} \chi_{v}+O(1)\left|\lambda_{m}\right| \chi_{m} \\
& =O(1) .
\end{aligned}
$$

In view of (2.1), (2.4), (2.5), (3.2), (3.3) and the boundedness of $\lambda_{n}$ (see [3])

$$
\begin{aligned}
\sum_{n=2}^{m+1} \frac{1}{n^{k}}\left|\varphi_{n} t_{n, 2}^{\alpha}\right|^{k} & \leq \sum_{n=2}^{m+1} \frac{\left|\varphi_{n}\right|^{k}}{n^{k} A_{n}^{\alpha}} \sum_{v=1}^{n-1} v^{k} A_{n-v-1}^{\alpha-1}\left|\lambda_{v}\right|^{k}\left|\Delta T_{v-1}\right|^{k}\left\{\sum_{v=1}^{n-1} \frac{A_{n-v-1}^{\alpha-1}}{A_{n}^{\alpha}}\right\}^{k-1} \\
& =O(1) \sum_{v=1}^{m} v^{k}\left|\lambda_{v}\right|^{k}\left|\Delta T_{v-1}\right|^{k} \sum_{n=v+1}^{m+1} \frac{\left|\varphi_{n}\right|^{k} A_{n-v-1}^{\alpha-1}}{n^{k} A_{n}^{\alpha}} \\
& =O(1) \sum_{v=1}^{m} v^{k}\left|\lambda_{v}\right|^{k}\left|\Delta T_{v-1}\right|^{k} \sum_{n=v+1}^{m+1} \frac{\left|\varphi_{n}\right|^{k}(n-v)^{\alpha-1}}{n^{k+\alpha}} \\
& =O(1) \sum_{v=1}^{m} v^{\epsilon}\left|\lambda_{v}\right|^{k}\left|\varphi_{v}\right|^{k}\left|\Delta T_{v-1}\right|^{k} \sum_{n=v+1}^{m+1} \frac{(n-v)^{\alpha-1}}{n^{\alpha+\epsilon}} \\
& =O(1) \sum_{v=1}^{m}\left|\lambda_{v}\right|^{k}\left|\varphi_{v}\right|^{k}\left|\Delta T_{v-1}\right|^{k} \\
& =O(1) \sum_{v=1}^{m}\left|\lambda_{v}\right| v^{1-k}\left(\frac{P_{v}}{p_{v}}\right)^{k-1}\left|\varphi_{v} \Delta T_{v-1}\right|^{k} \\
& =O(1) \sum_{v=1}^{m}\left|\lambda_{v}\right| v^{1-k \alpha}\left(\frac{P_{v}}{p_{v}}\right)^{k-1}\left|\varphi_{v} \Delta T_{v-1}\right|^{k} \\
& =O(1), \text { as in the case of } t_{n, 1}^{\alpha}
\end{aligned}
$$

$$
\begin{aligned}
\sum_{n=2}^{m+1} \frac{1}{n^{k}}\left|\varphi_{n} t_{n, 3}^{\alpha}\right|^{k} & \leq \sum_{n=2}^{m+1} \frac{\left|\varphi_{n}\right|^{k}}{n^{k}\left(A_{n}^{\alpha}\right)} \sum_{v=1}^{n-1}\left(\frac{P_{v}}{p_{v}}\right)^{k} A_{n-v-1}^{\alpha-1}\left|\lambda_{v}\right|^{k}\left|\Delta T_{v-1}\right|^{k}\left\{\sum_{v=1}^{n-1} \frac{A_{n-v-1}^{\alpha-1}}{A_{n}^{\alpha}}\right\}^{k-1} \\
& =O(1) \sum_{v=1}^{m}\left(\frac{P_{v}}{p_{v}}\right)^{k}\left|\lambda_{v}\right|^{k}\left|\Delta T_{v-1}\right|^{k} \sum_{n=v+1}^{m+1} \frac{\left|\varphi_{n}\right|^{k} \mid A_{n-v-1}^{\alpha-1}}{n^{k} A_{n}^{\alpha}}
\end{aligned}
$$




$$
\begin{aligned}
& =O(1) \sum_{v=1}^{m} v^{\epsilon-k}\left(\frac{P_{v}}{p_{v}}\right)^{k}\left|\lambda_{v}\right|^{k}\left|\varphi_{v}\right|^{k}\left|\Delta T_{v-1}\right|^{k} \sum_{n=v+1}^{m+1} \frac{(n-v)^{\alpha-1}}{n^{\alpha+\varepsilon}} \\
& =O(1) \sum_{v=1}^{m} v^{-k}\left(\frac{P_{v}}{p_{v}}\right)^{k}\left|\lambda_{v}\right|^{k}\left|\varphi_{v}\right|^{k}\left|\Delta T_{v-1}\right|^{k} \\
& =O(1) \sum_{v=1}^{m}\left|\lambda_{v}\right| v^{1-k}\left(\frac{P_{v}}{p_{v}}\right)^{k-1}\left|\varphi_{v} \Delta_{v-1}\right|^{k} \\
& =O(1), \text { as in the case of } t_{n, 2}^{\alpha}
\end{aligned}
$$

$$
\begin{aligned}
& \sum_{n=2}^{m+1} \frac{1}{n^{k}}\left|\varphi_{n} t_{n, 4}^{\alpha}\right|^{k} \\
\leq & \sum_{n=2}^{m+1} \frac{\left|\varphi_{n}\right|^{k}}{n\left(A_{n}^{\alpha}\right)^{k}} \sum_{v=1}^{n-1}(v+1)\left(\frac{P_{v}}{p_{v}}\right)^{k}\left(A_{n-v-1}^{\alpha-1}\right)^{k}\left|\Delta \lambda_{v}\right|\left|\Delta T_{v-1}\right|^{k}\left\{\frac{1}{n} \sum_{v=1}^{n-1}(v+1)\left|\Delta \lambda_{v}\right|\right\} \\
= & O(1) \sum_{v=1}^{m}\left(\frac{P_{v}}{p_{v}}\right)^{k}\left|\Delta \lambda_{v}\right|^{k}\left|\Delta T_{v-1}\right|^{k} \sum_{n=v+1}^{m+1} \frac{\left|\varphi_{n}\right|^{k} \mid\left(A_{n-v-1}^{\alpha-1}\right)^{k}}{n\left(A_{n}^{\alpha}\right)^{k}} \\
= & O(1) \sum_{v=1}^{m} v\left(\frac{P_{v}}{P_{v}}\right)^{k}\left|\Delta \lambda_{v}\right|\left|\Delta T_{v-1}\right|^{k} \sum_{n=v+1}^{m+1} \frac{\left|\varphi_{n}\right|^{k}(n-v)^{k \alpha-k}}{n^{1+k \alpha}} \\
= & O(1) \sum_{v=1}^{m} v^{1+\epsilon-k}\left(\frac{P_{v}}{p_{v}}\right)^{k}\left|\Delta \lambda_{v}\right|\left|\varphi_{v}\right|^{k}\left|\Delta T_{v-1}\right|^{k} \sum_{n=v+1}^{m+1} \frac{(n-v)^{k \alpha-k}}{n^{1+\epsilon+k \alpha-k}} \\
= & O(1) \sum_{v=1}^{m} v^{1-k}\left(\frac{P_{v}}{p_{v}}\right)^{k}\left|\Delta \lambda_{v}\right|\left|\varphi_{v}\right|^{k}\left|\Delta T_{v-1}\right|^{k} \\
= & O(1) \sum_{v=1}^{m}\left|\Delta \lambda_{v}\right| v^{2-k}\left(\frac{P_{v}}{p_{v}}\right)^{k-1}\left|\varphi_{v} \Delta T_{v-1}\right|^{k} \\
= & O(1) \sum_{v=1}^{m} v \beta_{v} v^{1-k \alpha}\left(\frac{P_{v}}{p_{v}}\right)^{k-1}\left|\varphi_{v} \Delta T_{v-1}\right|^{k} \\
= & O(1) \sum_{v=1}^{m}\left|\Delta\left(v \beta_{v}\right)\right| \chi_{v}+O(1) m \beta_{m} \chi_{m} \\
= & \sum_{v=1}^{m} \beta_{v}+\sum_{v=1}^{m}(v+1)\left|\Delta \beta_{v}\right| \chi_{v}+O(1) m \beta_{m} \chi_{m} \\
= &
\end{aligned}
$$


in veiw of (2.3), (2.5), (3.1), (3.2) and (3.3).

$$
\begin{aligned}
\sum_{n=1}^{m} \frac{1}{n^{k}}\left|\varphi_{n} t_{n, 5}^{\alpha}\right|^{k} & \leq \sum_{n=1}^{m} \frac{1}{\left(A_{n}^{\alpha}\right)^{k}}\left|\lambda_{n}\right|^{k}\left(\frac{P_{n}}{p_{n}}\right)^{k}\left|\varphi_{n} \Delta T_{n-1}\right|^{k} \\
& =O(1) \sum_{n=1}^{m}\left|\lambda_{n}\right| n^{1-k \alpha}\left(\frac{P_{n}}{p_{n}}\right)^{k-1}\left|\varphi_{n} \Delta T_{n-1}\right|^{k} \\
& =O(1) .
\end{aligned}
$$

This completes the proof of the Theorem.

\section{References}

[1] M. Balci, "Absolute $\varphi$-summability factors," Comm. Fac. Sci. Univ. Ankara, Ser. A1, 29 (1980), 63-68.

[2] H. Bor, "A note on two summability methods," Proc. Amer. Math. Soc., 98 (1986), 81-84.

[3] H. Bor, "On the absolute $\varphi$-summability factors of infinite series," Portugaliae Math. 45 (1988), 131-137.

[4] T. M. Flett, "On an extension of absolute summability and some theorems of Littlewood and Paley," Proc. Lond. Math. Soc., 7 (1957), 113-141.

[5] T. M. Flett, "Some more theorems concerning the absolute summability of Fourier series," Proc. Lond. Math. Soc., 8 (1958), 357-385.

[6] K. N. Mishra, \& R. S. L. Srivastava, "On absolute Cesàro summability factors of infinite series," Portugaliae Math., 42 (1983-1984), 53-61.

[7] W. T. Sulaiman, "Multipliers for the $\varphi-|C, \alpha|_{k}$ summability of infinite series," Pure Appl. Math. Sci., 31 (1990), 43-49.

Mosul University, Iraq. 